\title{
NOTE ON THE PRONUNCIATION OF MAYAN WORDS
}

Consonants may be pronounced as in English, with these exceptions: $j$ is like Spanish $j$, with the tongue farther back than for English $h ; l$ is like Welsh $l l$, with the tongue farther forward than for English $l ; q$ is like Hebrew qoph, with the tongue farther back than for English $k$; and $r$ usually has a single flap as in Spanish. Two other Mayan sounds are found in English but are spelled differently: $t z$ is like English $t s$ in "sets," and $x$ is like English $s h$. The glottal stop, indicated by', is the middle sound in the English expression " $m$ ' $m$," meaning "no"; when it follows another consonant, it is pronounced simultaneously with that consonant. Vowels are approximately like those of Spanish, except that doubled vowels are held longer, like the long vowels in Classical Greek. In some transcriptions from texts in the Mayan script, a vowel shown as doubled may have originally been followed by $h$ or ' rather than lengthened. Vowels are limited to five in the alphabetic documents of the colonial period, but most Mayan languages differentiate one or more of these five by means of lengthening or differences in tone. Stress is nearly always on the final syllable of a word. 
This page intentionally left blank 